\title{
PUENTES SOBRE LA TIERRA. ESTRATEGIAS DE BROKERS EN UNA RED DE VENTA DE TIERRA. PICHIDEGUA, CHILE, 1820-1845*
}

\author{
BRIDGES OVER LAND. STRATEGIES OF BROKERS IN A LAND SALE \\ NETWORK. PICHIDEGUA, CHILE, 1820-1845
}

\author{
Víctor Brangier ${ }^{* *}$, Alexis Matheu** y Alex Ovalle ${ }^{* * *}$
}

\begin{abstract}
El estudio problematiza el comportamiento de brokers en un circuito de venta de tierras en el valle de Pichidegua, zona central de Chile, entre 1820 y 1845 . Se analiza el accionar de estos actores ubicados en posiciones estratégicas de la red para conquistar esos espacios y obtener ventajas. Se abordaron 340 escrituras notariales de venta de tierras. Los nombres de los contratantes fueron procesados mediante el software UCINET para obtener una caracterización de la red, la identificación de los agujeros estructurales y dos medidas complementarias de centralidad provistas por el Análisis de Redes Sociales: intermediación y grado. Además se recurrió a tres catastros agrícolas levantados en la primera mitad del siglo XIX para aproximarse al perfil socioeconómico de los actores. Los resultados evidenciaron una correlación alta entre brokers y quienes registraron mayores lazos y capacidad de intermediación. Se concluye un comportamiento tipo de los brokers, registrados como agricultores de mediana rentabilidad, pactando con una alta cantidad sujetos, poco centrales y débilmente conectados a la estructura.
\end{abstract}

Palabras claves: Análisis de redes sociales, agujeros estructurales, UCINET, historia rural, Chile, siglo XIX.

This article problematizes the behavior of brokers in a circuit of land sales in the Pichidegua Valley, central zone of Chile, between 1820 and 1845. It analyzes the behavior of these actors located in strategic positions of the network to conquer those spaces and obtain advantages. 340 notarial documents of land sales were addressed. The names of the contractors were processed using the UCINET software to obtain a characterization of the network and the identification of the structural holes and two complementary measures of centrality provided by Social Networks Analysis: Betweenness and Degree. In addition, three agricultural cadastres of the first half of the nineteenth century were used to approach the socio-economic profile of the actors. The results showed a high correlation between brokers and those who registered greater ties and intermediation capacity. We conclude a typical behavior of the brokers, registered as farmers of medium profitability, agreeing with a high number of subjects, little central and weakly connected to the structure.

Key words: Social network analysis, structural holes, UCINET, rural history, Chile, 19th century.

\section{Introducción}

Existe una línea de estudios en perspectiva histórica que ha examinado la importancia de la posición de los actores en la red de relaciones para la obtención de ventajas. Desde ciertos lugares y no de otros en la trama relacional, sería posible lograr mejor acceso a recursos valiosos, como información, bienes, influencias, poder, etc. El acento ha estado puesto en la necesidad de ocupar posiciones de puente entre actores que de otro modo no se vincularían entre sí. Quienes cumplieran el papel de conectores se ubicarían en posiciones estratégicas de la red y el resto de actores dependería de ellos para integrarse a la estructura. Hace al menos cuatro décadas las monografías han apuntado en esta dirección. Se ha indagado la función que ha cumplido la posición de puente entre actores, subgrupos y estructuras sociales sin interconexión, como factor de la adquisición de ventajas en contextos gubernamentales, industriales y artísticos, como además entre pugnas de facciones familiares nobiliarias, académicas y mercantiles (respectivamente: Caro 1982; Dalzell 1987; Di Maggio 1992; Padgett 1993; Collins 1998; Greif 1989). Ronald Burt (2005) abordó una revisión amplia de esta discusión.

Los estudios que examinaron el funcionamiento de los espacios de las redes sociales con escasos vínculos y la presencia de los actores puentes, motivó al sociólogo norteamericano Ronald Burt

\footnotetext{
* Resultado Proyecto Fondecyt Iniciación No 11160130.

** Universidad Bernardo O’Higgins, Santiago, Chile. Correos electrónicos: vmbrangi@gmail.com; alexis.matheu@ubo.cl

*** Universidad de La Serena, La Serena, Chile. Correo electrónico: aovalle@ userena.cl
} 
a levantar la propuesta de los "agujeros estructurales". Un tipo de enfoque sensible a aquellos espacios internos de las redes identificados con vínculos débiles o inexistentes entre los actores. Para Burt (1992), los agujeros estructurales son microestructuras que constituyen una oportunidad de obtención de capital social para quienes tejan lazos entre los actores desconectados y el mundo circundante, sobre todo si sirve de nexo entre un grupo cerrado y otro. Burt aseguró que estos sujetos puentes, denominados brokers, se favorecen de la recepción y difusión de información variada y no redundante, toda vez que se comunican con sujetos portadores de novedades provenientes de distintas fuentes y escenarios sociales. En cada situación de comunicación, las propuestas e interpretaciones del broker serían valoradas por su carácter innovador y sugestivo. De todos modos, es una corriente que discute con la línea de estudios que sostiene que con el tiempo, la posición de actores en los agujeros estructurales se transforma en desventaja. Como sugirió James Coleman (1998), el capital social derivaría de la pertenencia de los sujetos a grupos cohesionados, donde circulan confianzas, información fidedigna y solidaridades ${ }^{1}$.

El mismo Ronald Burt indicó que la idea de terceros que se benefician de la desconexión de sus pares no es nueva. Ya la había subrayado a principios de siglo XX el sociólogo alemán Georg Simmel con su teoría del tertius gaudens, o el tercero que se beneficia de la intermediación de otros dos desconectados (Tortoriello and Krackhardt 2010). Por la misma época, Joseph Schumpeter (1934) visualizó la ventaja de líderes empresariales que unían elementos de esferas productivas disímiles. Más tarde, Robert Merton (1957) destacó la importancia que tiene la vinculación de subgrupos en la difusión de ideas políticas. Por supuesto el enfoque tomó fuerza desde la década de 1960 con los estudios del "mundo pequeño" de Stanley Milgram (1969) y la dependencia de los intermediadores estratégicos para conectar, en pocos pasos, a cada actor con el resto del mundo. También, y cómo sintetizó Burt (2004), esta vertiente se desarrolló desde la influyente tesis de la "fortaleza de los lazos débiles", de Mark Granovetter (1973), quien puntualizó que la sobrevivencia de un grupo depende de sus conexiones con redes exógenas, obteniendo información nueva y no redundante. Más recientemente, las indagaciones han intentado poner a prueba y en distintos contextos empíricos las propuestas de Ronald Burt acerca de las ventajas obtenidas por los brokers que gestionan su propia posición en medio de agujeros estructurales. Por ejemplo, Buskens and Van de Rijt (2008) experimentaron desde la teoría del juego la distribución de beneficios entre distintos brokers y las subredes que emergían desde su interacción. Muy cerca de este ejercicio, varias monografías han intentado dilucidar la gestión que emprenden los brokers respecto de su posición de intermediadores estratégicos en los agujeros estructurales (Goyal and Vega-Redondo 2007; Kleinberg, et al. 2008; Lou \& Tang 2013; Fagan 2017). De todos modos, para el mismo Ronald Burt, la idea de actores de la red insertos en agujeros estructurales conlleva inherente una acción de gestión de esa condición. Ese agenciamiento estaría orientado a la intermediación de sujetos vinculados al broker. Burt denominó esa acción intrínseca como brokerage (Peeples and Haas 2013).

Este estudio deriva de la última vertiente de la discusión respecto de los agujeros estructurales y problematiza el comportamiento de los brokers en medio de estos espacios de las redes sociales. Para ello, la investigación se centrará específicamente en una red de relaciones por venta de tierras, en un valle de la zona central de Chile durante la primera mitad del siglo XIX. Se trató del valle de Pichidegua, sector de 77 kilómetros cuadrados y distante a 116 kilómetros al sur de Santiago. Integró la provincia de Colchagua, creada tras las leyes federales de 1826 y se extendió a lo largo de una región que experimentó el predominio de la gran hacienda cerealera desde el siglo XVIII (Mellafe 2004: 107-108)2.

La literatura que ha abordado la constitución de la propiedad de la tierra en la región central y sur del país, ha tendido a visualizar la concentración sin contrapeso del bien raíz en manos de los conquistadores (mediante recepción de mercedes de tierras) y, luego, desde el periodo colonial, de los latifundistas quienes habrían movido los resortes de la economía y el comercio agrícola (Mellafe 2004; Cáceres 2007; Muñoz 2008; Llorca et al. 2017: 612). En contraposición, la historiografía social rural ha previsto que las economías campesinas supieron mantener su autonomía y, desde la resistencia a la gran hacienda triguera, sostuvieron la economía local (Romano1965; Salazar y Pinto 2002: 97-99). De ese modo, la discusión ha tendido a mantener una perspectiva dicotómica, omitiendo el examen de la circulación de tierras entre distintos 
estratos y sobre todo, el funcionamiento de redes sociales transversales emanadas de esos vínculos. Esta omisión se vuelve más aguda para el análisis de la primera mitad del siglo XIX, periodo en el que existe un menor abordaje monográfico (Llorca, et al. 2017). En este sentido, el artículo busca caracterizar la constitución de una red por venta de tierras en Pichidegua en esta época. Además, se pretende analizar el comportamiento tipo de los brokers en los agujeros estructurales de la red. Por último se procura explicar dicho comportamiento a partir de su relación con dos dimensiones de centralidad de la red: el grado y la intermediación, nociones que se definirán en el apartado siguiente.

Como línea hipotética se plantea que los brokers presentes en la red de venta de tierras en el valle de Pichidegua en este periodo, fueron actores que alcanzaron esa función mediante una estrategia específica: estos sujetos privilegiaron pactar más transacciones que el promedio de quienes integraron la red. Además, tendieron a vincularse con sujetos que no presentaron ventas entre sí y, en general, eran advenedizos en las prácticas de venta (tenían pocos lazos y, por tanto, no ocupaban una posición de centralidad en la red). De ese modo, los brokers construían sus propios agujeros estructurales para lograr la dependencia de su vecindario dentro de la red.

El estudio se extiende entre 1820 y 1845 . Antes de esa fecha, esta área fue escenario predilecto de cruentas batallas durante la coyuntura de independencia, alterando profundamente los términos de la economía agrícola-ganadera y la posesión y transferencia de la tierra (Rector 1985). El periodo y espacio escogido corresponden a un escenario de reconfiguración de la tenencia y traspaso de tierra, previo al "auge triguero", que se extendió desde inicios de la década de 1850. Desde entonces, la presión derivada por el alza en el valor de la tierra y de los bienes agrícolas y ganaderos abrió un nuevo escenario en las transferencias de este bien raíz y las relaciones sociales vinculadas (Cariola y Sunkel 1982; Salazar y Pinto 2002).

\section{Método: análisis de redes sociales e identificación de agujeros estructurales}

Desde la perspectiva de los agujeros estructurales de Ronald Burt, el estudio examinó la formación de una red social por venta de tierras a partir de 340 documentos notariales celebrados en el valle de Pichidegua entre 1820 y 1845. El material corresponde a escrituras elaboradas por los jueces legos de este sector, particularmente por los inspectores de los distritos de Almahue y de Pichidegua ${ }^{3}$. Por lo general, cada documento era redactado en presencia de compradores y vendedores y el inspector declaraba actuar frente a testigos ante ausencia de notarios. Las escrituras contenían el nombre de los contratantes, información que ha permitido su registro y posterior procesamiento por el software UCINET para la estructuración, diseño y medición de la red social. Los 340 documentos examinados corresponden a la totalidad de contratos de venta de tierras de los distritos seleccionados que conserva el Fondo de Notarios de Rengo, distribuidos entre el Archivo Nacional Histórico de Chile [ANH] (volumen 2) y el Archivo Nacional de la Administración [ARNAD] (volumen 26) ${ }^{4}$.

El escrutinio de los agujeros estructurales de esta red social se efectuó por medio de la medición de la variable Efficient Size. Es una medida que identifica estas estructuras mediante la detección de nodos (puntos o actores de la red) insertos en vecindarios de la red con baja conexión. La variable descubre aquellos actores vinculados a nodos no relacionados entre ellos (Burt 1992; Latora, et al. 2013: 751). A mayor puntuación en los resultados, mayor inserción del actor en un agujero estructural. Por el contrario, a menor puntuación, mayor vinculación con nodos vinculados entre ellos, lo que indicaría la pertenencia a un grupo cohesionado. Los resultados de esta medición se cotejarán con otras dos variables utilizadas en la dimensión de centralidad del Análisis de Redes Sociales: Degree y Betweenness. El primero de ellos cuantifica la cantidad de lazos registrados por cada nodo y la segunda la cantidad de veces en que un nodo figura entre las rutas geodésicas (los caminos más cortos y transitando por menos cantidad de nodos) entre otros dos nodos de la red (Borgatti, Everett and Johnson 2013). Las cifras obtenidas por Betweenness son directamente proporcional a la capacidad de intermediación. Es decir, a mayor numeración obtenida por el nodo, mayor cantidad de veces se encuentra en la ruta geodésica entre otros dos nodos de la red. En síntesis, la variable Degree explica el grado de conexión que un nodo tiene en la red (contabilizando la cantidad de vínculos), mientras que Betweenness permite medir la posición estratégica de los actores instalados en 
medio de los caminos más probables que seguiría un nodo para alcanzar un tercero.

Para analizar el comportamiento de los brokers posicionados en agujeros estructurales y su nexo con las medidas de centralidad, se estudió la correlación entre las tres variables, recurriendo a los coeficientes de relación. El instrumento mide el grado en que las variables se relacionan y tienden a cambios de manera simultánea. Se trata de coeficientes que miden la fuerza y la dirección de estas relaciones ${ }^{5}$.

\section{Resultados: los brokers de la tierra y la búsqueda de centralidad}

El procesamiento de los datos por medio de UCINET dio como resultado un grafo de red de 340 relaciones y de 347 nodos. 211 de estos $(60,8 \%)$ se interconectaron a lo largo de un componente principal, sin evidencias de contratos de ventas con el 39,2\% restante. La Figura 1 expresa la fisonomía de la estructura, integrada por un componente principal de gran volumen, circunvalado a su vez por varios actores débilmente conectados entre sí.

La indagación de la red resalta la existencia de pocos actores centrales (escasos nodos con altas conexiones y capacidad de intermediación), como además, una baja cantidad de agujeros estructurales y de brokers. Así lo demuestra el análisis de las variables Efficient Size, Degree y Betweenness. La Tabla 1 ofrece los resultados de la cantidad de nodos (Q) por valor obtenido en las dos primeras variables. La Tabla 2 entrega el resultado del mismo ejercicio en la tercera variable. La presentación se realiza de manera separada, pues las cifras obtenidas en la medición de Betweenness son altas en comparación de las que arroja UCINET para Efficient Size y para Degree. Estas últimas oscilan entre 1 y 16 puntos, mientras que aquella entre 0 y $8.033,2$ puntos. Para facilitar la lectura de los resultados en Betweenness se han reducido las cifras a 10 rangos. Se ha resaltado la primera de las cifras (0) y una intermedia (209), pues han concentrado una cantidad de nodos que resulta inusual al resto de los rangos.

Los resultados de la Tabla 1 demuestran que la gran mayoría de los nodos obtuvo el mínimo posible en las cifras de las dos variables $(224 \mathrm{y}$ 220 del total de 347 nodos de la red, en Efficient Size y Degree, respectivamente) y que hubo una disminución progresiva en la medida que los valores aumentaban, hasta llegar a la relación 1:1 entre valor de variable y cantidad de nodos en las últimas filas. Los datos indican que en la red de venta de

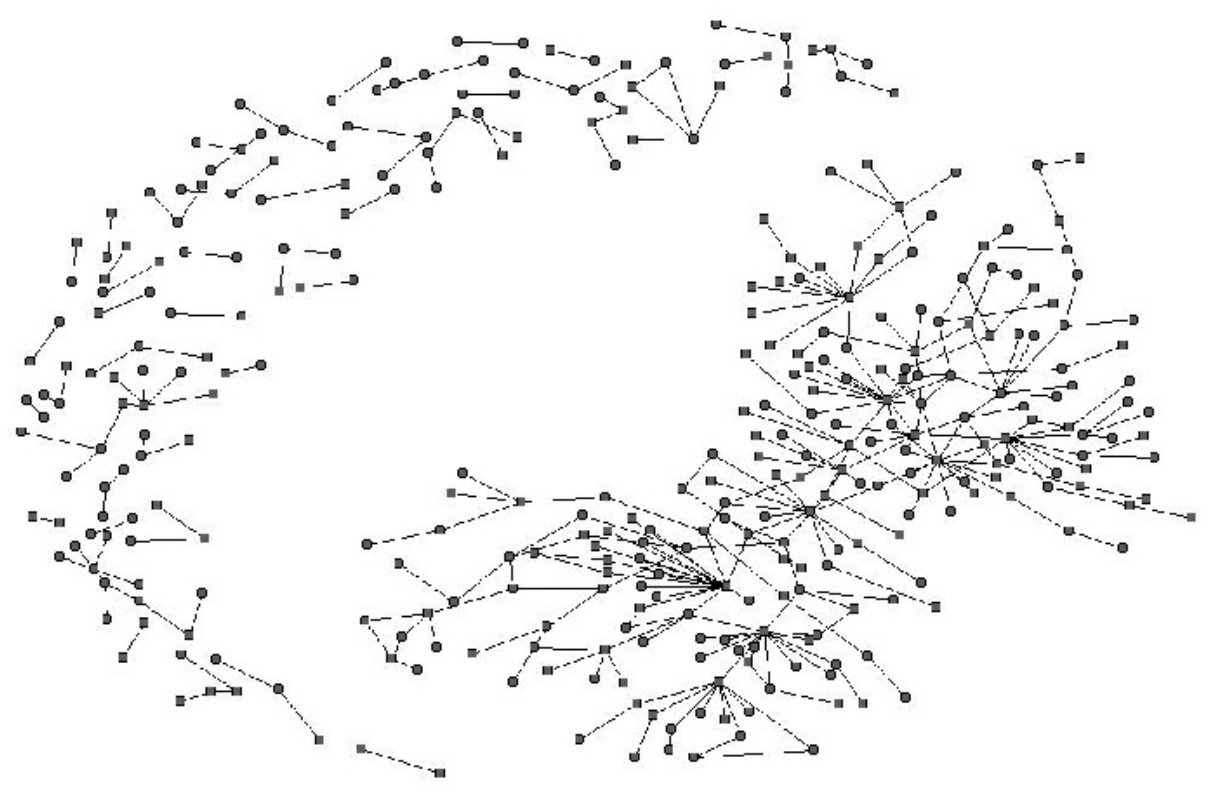

Figura 1. Red de venta de tierras en Pichidegua, 1820-1845.

Fuente: 340 escrituras de venta de tierras. ANH, Fondo Notarios de Rengo, volumen 2; ARNAD, Fondo Notarios de Rengo, volumen 26. Datos procesados mediante software UCINET, versión 6.658. 
Tabla 1. Cantidad de nodos por valor en variables Efficient Size y Degree.

\begin{tabular}{|c|c|c|}
\hline \multirow{2}{*}{ Valor } & \multicolumn{2}{|c|}{ Q } \\
\hline & Efficient Size & Degree \\
\hline 1 & 224 & 220 \\
\hline 2 & 74 & 78 \\
\hline 2,3 & 3 & - \\
\hline 3 & 16 & 19 \\
\hline 3,7 & 1 & - \\
\hline 4 & 8 & 10 \\
\hline 4,6 & 1 & - \\
\hline 5 & 5 & 6 \\
\hline 6 & 3 & 2 \\
\hline 6,6 & 1 & - \\
\hline 7 & 1 & 2 \\
\hline 7,8 & 1 & - \\
\hline 8 & 1 & 2 \\
\hline 9 & 1 & 1 \\
\hline 9,8 & 1 & - \\
\hline 10 & - & 1 \\
\hline 11 & 1 & 1 \\
\hline 12 & 1 & 1 \\
\hline 13 & - & - \\
\hline 13,7 & 1 & - \\
\hline 13,8 & 1 & - \\
\hline 14 & - & 2 \\
\hline 14,8 & 1 & - \\
\hline 15 & - & 1 \\
\hline 16 & 1 & 1 \\
\hline
\end{tabular}

Fuente: 340 escrituras de venta de tierras. ANH, Fondo Notarios de Rengo, volumen 2; ARNAD, Fondo Notarios de Rengo, volumen 26. Datos procesados mediante software UCINET, versión 6.658 .

tierras en este sector, el grueso de los contratantes no se vinculó a sujetos desconectados entre sí y, por tanto, los pocos agujeros estructurales existentes estuvieron monopolizados por pocos brokers. Este hecho se expresa en la concentración de nodos en los primeros valores más bajos de Efficient Size y que desde el valor 5 al 16 se encuentran solo 20 sujetos. Es decir, en los dos últimos tercios de los valores de la tabla se encontró solo el 5,7\% de los nodos. Del mismo modo, los actores solían integrar la estructura relacionándose con una a tres personas como máximo. Los nodos que se vincularon a más de cuatro fueron la minoría (lo hicieron 20 de los 347 nodos: 5,7\%).

Por su parte, la Tabla 2 informa resultados similares a la Tabla 1. Quienes lograron posicionarse como intermediadores estratégicos (instalados en las rutas geodésicas de los caminos de la red) fueron escasos y la mayoría simplemente no se ubicó
Tabla 2. Cantidad de nodos por valor en variable Betweenness.

\begin{tabular}{cc}
\hline Rangos de Valor & Q \\
\hline 0 & 222 \\
$0,5-1$ & 16 \\
$2-12,5$ & 14 \\
$3-207$ & 15 \\
209 & 20 \\
$209,5-416$ & 14 \\
$417-827$ & 12 \\
$829-1992,1$ & 12 \\
$2229,5-3388,6$ & 12 \\
$3696,1-8033,2$ & 10 \\
\hline
\end{tabular}

Fuente: 340 escrituras de venta de tierras. ANH, Fondo Notarios de Rengo, volumen 2; ARNAD, Fondo Notarios de Rengo, volumen 26. Datos procesados mediante software UCINET, versión 6.658 .

en ningún camino geodésico (en el valor cero se concentraron 222 de los 347 nodos, equivalente al $63,9 \%$ ). El último rango se vincula a los valores de intermediación más altos de la red y allí se reconocieron solo 10 nodos (2,8\% del total).

El cotejo de las tres variables -considerando como eje del escrutinio el indicador Efficient Sizearrojó como resultado principal una alta correlación entre ellas. Es decir, los nodos que lograron posicionarse en agujeros estructurales de mayor envergadura, fueron a la vez los actores con mayores lazos y cumplieron el papel de intermediadores en la red. La Tabla 3 presenta una selección de los nombres de los 20 primeros actores (Top 20), ordenados de mayor a menor, registrados con los mayores valores en cada una de las variables.

La Tabla 3 expone a 27 contratantes en total. 13 de ellos figuraron en las tres columnas, siete en dos y otros siete solo en una. En otros términos, prácticamente la mitad $(48,14 \%)$ de los 20 nodos que registraron mayores índices en una variable, estuvieron presentes en la lista Top 20 de las otras dos, develando la correlación elevada entre las tres mediciones. De los 20 actores reconocidos en la lista Efficient Size, 18 coinciden con la lista Degree, expresando una coincidencia de $90 \%$. De modo similar, el cotejo entre la lista Efficient Size y los nodos registrados en el Top 20 de la variable Betweenness, arrojó una equivalencia del $65 \%$ (compartieron 13 nodos).

Si se trasciende la selección de los 20 primeros índices en cada una de las variables y se amplía el 
Tabla 3. Top 20 Effient size, Degree y Betweenness.

\begin{tabular}{lclllc}
\hline \multicolumn{1}{c}{ Nodo } & Eff. size & \multicolumn{1}{c}{ Nodo } & Degree & \multicolumn{1}{c}{ Nodo } & Betweenness \\
\hline Vicente Echenique & 16 & Bartolo Catalan & 20 & Martin Galvez & 8033,258 \\
Luis Tolorza & 14,867 & Vicente Echenique & 19 & Juan Ortiz & 7745,842 \\
Francisco Silva & 13,867 & Francisco Silva & 15 & Bartolo Catalan & 7432,1 \\
Bartolo Catalan & 13,833 & Luis Tolorza & 15 & Jose Maria Padilla & 5586,35 \\
Martin Galvez & 12 & Martin Galvez & 12 & Luis Tolorza & 5505,933 \\
Justo Sanchez & 11 & Bernabel Trujillo & 11 & Manuel Donoso & 4780,483 \\
Bernabel Trujillo & 9,773 & Justo Sanchez & 11 & Juan Jose Tolorza & 4680,633 \\
Jose Zenon & 9 & Antonio Espinoza & 9 & Dominga Gutierrez & 4358,292 \\
Antonio Espinoza & 8 & Jose Zenon & 9 & Vicente Echenique & 4218,2 \\
Jose Maria Padilla & 7,87 & Dominga Gutierrez & 8 & Francisco Silva & 3696,108 \\
Dominga Gutierrez & 7 & Jose Maria Padilla & 8 & Juan Jose Romo & 3388,608 \\
Juan Espinoza & 6,688 & Manuel Donoso & 8 & Valentina Sanchez & 3281 \\
Juan Ortiz & 6 & Juan Espinoza & 7 & Bernabel Trujillo & 3246,433 \\
Manuel Donoso & 6 & Juan Ortiz & 6 & Justo Sanchez & 3207,5 \\
Jose Maria Rojas & 5 & Jose Maria Rojas & 5 & Agustin Bozo & 3112,5 \\
Manuel Ibarra & 5 & Manuel Ibarra & 5 & Jose Diaz & 2878,4 \\
Maria Ibarra & 5 & Maria Espinoza & 5 & Emiliana Rodriguez & 2524,65 \\
Prudencio Barrios & 5 & Maria Ibarra & 5 & Jose Zenon & 2436,5 \\
Vicente Fernandez & 5 & Prudencio Barrios & 5 & Antonio Espinoza & 2354,333 \\
Maria Espinoza & 4,7 & Tomas Padilla & 5 & Tomas Padilla & 2342,633 \\
\hline
\end{tabular}

Fuente: 340 escrituras de venta de tierras. ANH, Fondo Notarios de Rengo, volumen 2; ARNAD, Fondo Notarios de Rengo, volumen 26. Datos procesados mediante software UCINET, versión 6.658.

Tabla 4. Resultados de la Correlación de Spearman: Efficient size, Degree y Betweenness.

\begin{tabular}{|c|c|c|c|c|c|}
\hline \multicolumn{3}{|l|}{ Correlaciones } & \multirow{2}{*}{$\begin{array}{c}\text { Degree } \\
1,000\end{array}$} & \multirow{3}{*}{$\begin{array}{c}\text { Betweenness } \\
931\left(^{* *}\right) \\
, 000\end{array}$} & \multirow{2}{*}{$\begin{array}{c}\text { Efficient Size } \\
\left., 950{ }^{* *}\right)\end{array}$} \\
\hline & & Coeficiente de correlación & & & \\
\hline & Degree & Sig. (bilateral) & & &, 000 \\
\hline & & $\mathrm{N}$ & 347 & 347 & 347 \\
\hline \multirow[t]{6}{*}{ Rho(*) de Spearman } & & Coeficiente de correlación & ,931(**) & 1,000 & ,980(**) \\
\hline & Betweenness & Sig. (bilateral) &, 000 & &, 000 \\
\hline & & $\mathrm{N}$ & 347 & 347 & 347 \\
\hline & & Coeficiente de correlación &, $\left.950{ }^{* *}\right)$ &, $980\left(^{* *}\right)$ & 1,000 \\
\hline & Efficient Size & Sig. (bilateral) &, 000 &, 000 & \\
\hline & & $\mathrm{N}$ & 347 & 347 & 347 \\
\hline
\end{tabular}

(*) Coeficiente de Correlación.

(**) La correlación es significativa en el nivel 0,01 (2 colas).

Fuente: Resultados de variables obtenidas desde 340 escrituras de venta de tierras. ANH, Fondo Notarios de Rengo, volumen 2; ARNAD, Fondo Notarios de Rengo, volumen 26. Datos procesados mediante software UCINET, versión 6.658.

examen hacia los resultados íntegros, es posible detectar un alto grado de correlación entre ellos. La Tabla 4 expone los resultados tras la aplicación del Test de Correlación de Spearman acerca de los resultados de las tres variables.

$\mathrm{Al}$ observar las correlaciones entre las variables de manera binaria (de a dos) es posible determinar que el coeficiente de correlación en todos los casos es cercano a 1 , por lo que existe una relación alta 6 .
Por tanto, se puede afirmar que en esta red de venta de tierras la variable Efficient Size se relaciona estrechamente con las variables Degree y Betweenness. Como demuestra la Tabla 4, el coeficiente Rho de Spearman es de ,950 y ,980 evidenciando una correlación positiva alta. El resultado además se corrobora en el examen del nivel de significancia que, en este caso, es menor que ,005 (inclusive inferior a 0,01$)^{7}$. Ambas lecturas permiten visualizar 
la relación significativamente estrecha entre las tres variables.

\section{Discusión: sectores medios y gestión de los agujeros estructurales}

Los resultados evidenciaron una concentración de nodos en las cifras más bajas en cada variable. En los 25 años de prácticas de venta de tierras en el valle de Pichidegua, la mayoría de los contratantes frecuentaron pactar transferencias con un máximo de tres sujetos. Además, este patrón develó que se trató de contratos entre actores insertos en la red, lo que explica la baja presencia de agujeros estructurales y de brokers. Así entonces, el primer dato derivado de los resultados fue la concentración de los papeles de brokers y de intermediadores en pocos nodos. Del mismo modo, resaltó la excepcionalidad de quienes se vincularon con cuatro o más actores.

El segundo dato que se extrajo de los resultados fue la alta relación entre las variables. El análisis permite considerar que los nodos que monopolizaron las posiciones de brokers y de intermediadores, así también como quienes transaron ventas más veces, tendieron a ser los mismos actores. Es una primera aproximación que apunta a inferir que quienes buscaron contratar con una mayor cantidad de actores, privilegiaron hacerlo con sujetos desconectados entre sí y ligados débilmente a la red. En estos casos, hubo una predilección por buscar a los sujetos menos centrales para conectarse, como también a quienes no solían vender ni comprar tierras en este sector. El hecho se ratifica con la correlación entre las variables Eficcient size y Degree con Betweenness, indicando que los actores más vinculados se constituían automáticamente en brokers, privilegiando la unión con sujetos desconectados (sin participación en ventas). De ese modo, incrementaban su capacidad de intermediación entre estos nodos y el resto de la red. Este comportamiento estratégico fue asumido por pocos contratantes (las Tablas 1 a 3 resaltaron a los 20 principales, equivalentes al 5,7\% del total de participantes en la red), quienes se convirtieron en los brokers de los agujeros estructurales que se registraron excepcionalmente.

El 5,7\% de los sujetos de la red emprendieron una táctica distinta de compra y venta de tierras en esta localidad durante la primera mitad del siglo XIX: aspiraron transar tierras con muchos sujetos, privilegiando que estos no tuvieran antecedentes de traspaso entre sí y que, en general, tuvieran baja conexión a la red. El dato ofrece una oportunidad para retomar la discusión respecto de la constitución de la propiedad de la tierra en la zona centro sur del país. En específico, como se indicó más arriba, para arrojar luces acerca de uno de los aspectos menos indagados: la circulación de tierras entre estratos de distinto origen y las redes sociales que de allí surgieron. En otro estudio, el de las prácticas de transferencia de tierras en este sector-aunque trabajando con una base de datos menor y en un periodo más acotado-, fue posible descubrir que los nodos centrales de la red fueron actores que registraban rentabilidad mediana de sus tierras. Los agricultores catalogados entonces como "sectores medios" habrían logrado posicionarse en caminos cruciales de la red, dominando la intermediación entre el resto de los participantes. Las conclusiones entonces dialogaron con una historiografía rural que había puesto el acento en la hegemonía de los grandes hacendados en la constitución de la propiedad de la tierra y en las relaciones sociales que de allí se derivaron (Brangier et al. 2019a). Los resultados de esta investigación avanzan un grado en este debate examinando el comportamiento de los actores en otra de las posiciones estratégicas de la red: los agujeros estructurales. Así, el escrutinio de los 20 principales brokers de la red, en los tres catastros agrícolas levantados durante la época, posibilita aproximarse a su atributo socioeconómico ${ }^{8}$. $\mathrm{La}$ Tabla 5 exhibe el resultado de esta búsqueda puntualizando el nombre del actor, el año del catastro en el que fue encontrado, la localidad donde registró propiedad y la renta anual del predio.

La Tabla 5 evidencia que entre los 20 principales brokers de esta red, se encontraron ocho agricultores (menos de la mitad) que no alcanzaron la rentabilidad mínima para ser gravados. El hallazgo se vuelve significativo si se considera que la mayoría amplia de los nombres de los actores de la red no se encontraron en los catastros, siendo un circuito protagonizado por pequeños propietarios ${ }^{9}$. La Tabla 5 , entonces, indica que los medianos y grandes propietarios (escasos en el conjunto) se concentraron en las posiciones estratégicas de la red: en este caso, como brokers en agujeros estructurales ${ }^{10}$. Si se estipula la cifra de 100 pesos de rentabilidad como límite entre las categorías de mediano y de gran propietario, de los 20 brokers identificados, 10 ingresaron a la primera de ellas y cinco a la segunda. Tres nodos figuraron en ambas categorías, desplazándose de medianos a grandes propietarios en el lapso de 
Tabla 5. Top 20 brokers de la red de venta de Pichidegua, en tres catastros agrícolas.

\begin{tabular}{lccc}
\hline \multicolumn{1}{c}{ Broker } & Catastro agrícola & Localidad & Renta anual (pesos) \\
\hline Vicente Echenique & $1833 / 34$ & Pichidegua & 100 \\
Luis Tolorza & $1833 / 34$ & Pichidegua & 18,6 \\
Francisco Silva & 1852 & Pencahue & 150 \\
Bartolo Catalan & $1833 / 34$ & Pichidegua & 50 \\
Martin Galvez & 1852 & El Olivar & 200 \\
Justo Sanchez & 1855 & Tinguiririca & 28 \\
Bernabel Trujillo & - & - & - \\
Jose Zenon & - & - & - \\
Antonio Espinoza & $1833 / 34$ & Pichidegua & 25 \\
Jose Maria Padilla & 1855 & Pichidegua & 75 \\
Dominga Gutierrez & 1834 & Pichidegua & 25 \\
Juan Espinoza & 1852 & Pichidegua & 125 \\
Juan Ortiz & - & - & - \\
& - & - & - \\
Manuel Donoso & 1852 & Pencahue & 25 \\
& 1855 & Pencahue & 60 \\
Jose Maria Rojas & $1833 / 34$ & Pichidegua & 1000 \\
Manuel Ibarra & 1852 & Pichidegua & 2075 \\
Maria Ibarra & 1855 & Pichidegua & - \\
Prudencio Barrios & - & - & - \\
Vicente Fernandez & - & - & - \\
Maria Espinoza & $1833 / 1834$ & Pichidegua & - \\
\hline
\end{tabular}

Fuente: 340 escrituras de venta de tierras. ANH, Fondo Notarios de Rengo, volumen 2; ARNAD, Fondo Notarios de Rengo, volumen 26; ANH, "Catastro de la Provincia de Colchagua", 1834, Fondo de Contaduría Mayor, segunda serie, volumen 3742; Oficina Central de Estadística (1855a, 1855b).

elaboración entre un catastro y otro (los casos de Martín Gálvez, José María Padilla y de Prudencio Barrios). Se trató, entonces, de una red cuyos indicadores de centralidad (grado e intermediación) y de agujeros estructurales, fue protagonizada por actores medios, más que por grandes hacendados como ha sido el lugar común.

El atributo socioeconómico de estos nodos y el examen de su comportamiento, adicionalmente otorgan pistas para dialogar con los análisis respecto del comportamiento de los brokers y su gestión de las intermediaciones. Como se reseñó más arriba, los estudios recientes en Análisis de Redes Sociales han partido desde las propuestas de los agujeros estructurales de Ronald Burt, para evaluar el modo en que los brokers gestionan estas subestructuras de la red. En esta línea, estos resultados develan las estrategias emprendidas principalmente por sectores medios del valle de Pichidegua, para posicionarse en agujeros estructurales de la red de ventas de tierras y obtener ventajas de su posición de broker. El comportamiento se caracterizó por la inserción en una red de actores con distintos atributos socioeconómicos. Allí, y en una época de recomposición de la propiedad de la tierra y de la economía agroganadera, adquirieron tierras, transando con hacendados y principalmente con pequeños propietarios. Sobre todo, la estrategia de los sectores medios fue la conquista de agujeros estructurales de la red, vinculándose con quienes no tenían antecedentes de traspasos recíprocos y que también se encontraban débilmente vinculados al conjunto de la red. En alguna medida, estos sectores medios intentaron conquistar la dependencia de una mayor cantidad de actores en los circuitos de transacción de tierras ${ }^{11}$.

\section{Conclusión}

El artículo consideró la discusión pertinente a las ventajas de los actores que se posicionan en agujeros estructurales en redes sociales determinadas. 
Desde la vertiente analítica de la gestión que esos nodos intentan para conquistar y navegar esas estructuras, se problematizó el comportamiento de brokers en una red de venta de tierras en un valle de la zona central de Chile entre 1820 y 1845 , época de recomposición de las prácticas agroganaderas y del acceso a este bien raíz. Se buscó caracterizar esta red, como además analizar el comportamiento de los brokers registrados en agujeros estructurales. En última instancia, la investigación pretendió explicar esas conductas desde la relación de la condición de puentes estratégicos de la red con dos dimensiones de centralidad: el grado y la intermediación.

Mediante el análisis de 340 escrituras de venta de tierras, y con la ayuda del software UCINET, fue posible la caracterización de esta red y el registro de un componente principal a lo largo del que circuló la tierra en estas dos décadas y media. Esta subestructura excluyó al $40 \%$ de los actores registrados en las escrituras. Con UCINET se logró además la identificación de los agujeros estructurales, de los actores insertos en estos nichos y la medición de las variables de grado y de intermediación. Por medio de la aplicación del Test de Correlación de Spearman se evidenció la correlación alta entre las tres variables. De ese modo, se logró una aproximación a una de las conductas más significativas de los brokers del estudio: su inclinación por transar con varios sujetos, privilegiando que estos estuvieran desconectados entre sí y de la red, como además que no tuvieran antecedentes de transacciones. El cumplimento de los objetivos y los resultados obtenidos permitieron corroborar la línea hipotética respecto de la concentración de esfuerzos y recursos de los brokers por construir sus propios agujeros estructurales con tal de conquistar la dependencia de su vecindario en la red y posiciones claves de intermediación.
La investigación complementó los resultados de monografías anteriores respecto de la interrelación entre actores de estratos socioeconómicos diversos. Sobre todo, la predominancia que tuvieron en esta red los propietarios de mediana rentabilidad. En estas páginas se avanzó un grado en la aproximación a las estrategias de estos actores medios que, en un periodo de recomposición en las prácticas de acceso y uso de tierras, intentaron posicionarse en espacios claves de los circuitos de transferencias del valle de Pichidegua. Los resultados instan a profundizar en las conductas tipo de los nodos que escalaron hasta vértices estratégicos de esta red, más allá de las variables consideradas aquí, ampliando la mirada en relación con la conquista de Capital Social, según indica la teoría de Ronald Burt de los agujeros estructurales. Pero, asimismo, conviene complementar con el examen del resultado de este comportamiento en un periodo mayor, pertinente a la experiencia de cada uno de los brokers identificados, para evaluar la sustentabilidad de las estrategias. En este sentido, el artículo exhorta a reducir la escala de observación para pesquisar la trayectoria de estos actores y la relación sujeto-estructura de red, recurriendo al estudio de caso y a la perspectiva microanalítica ${ }^{12}$.

\section{Agradecimientos}

Esta investigación se desarrolló en el marco del proyecto de investigación CONICYT/FONDECYT de Iniciación $\mathrm{N}^{\circ} 11160130$, periodo 2016-2018; Los coautores agradecen a los evaluadores por la revisión de la primera versión y por los comentarios y observaciones que permitieron enriquecer el manuscrito. También agradecen el espacio brindado por el proyecto HISONET (History and Social Network): http://centroestudioshistoricos. ubo.cl/hisonet/, en cuyos talleres se discutieron los resultados y conclusiones de esta investigación.

\section{Referencias Citadas}

Álvarez, L.

2014 Catastro de propiedad en Chile: orígenes y evolución. Tesis para optar al grado de Doctor. Programa de Doctorado. Geografía, Planificación Territorial y Gestión Ambiental, Universidad de Barcelona, Barcelona.

Avner, G.

1989 "Reputation and Coalition in medieval trade: evidence on the Maghribi traders". Journal of Economic History 49:857-852.
Borgatti, S.; Everett, M. \& Johnson, J.

2013 Analyzing Social Network. Sage, New York.

Brangier, V.

2019a "Análisis de Redes Sociales y venta de tierras: un estudio de caso de Chile en perspectiva histórica". Población y Sociedad 1, 26, en prensa.

Brangier, V.

2019b "Intermediación y Grado en una red de venta de tierras Valle de Pichidegua, Chile, 1820-1835". Revue Caravelle 111 , en prensa. 
Brangier, V.; Díaz, A.; Morong, G.

2018a "Acusaciones contra jueces legos ante jueces de Letras: uso social del avance de la justicia letrada. Zona centro-sur de Chile, 1824-1875". História Unisinos 22, 1:75-87.

Brangier, V.; Silva, M.; Muñoz, G.

2018b "Selección de escrituras de transferencia de tierra en Pichidegua, Chile, 1814-1836. Extraídas del expediente 'Inbentario de los bienes del finado Dn Vicente Echeñique. 1842"”. Historia y Justicia 11, 404-426.

Burt, R.

1992 Structural Holes. The Social Structure of Competition. Harvard University Press, Cambridge.

Burt, R.

2004 "Structural holes and good ideas". American Journal of Sociology 110, 2:349-399.

Burt, R.

2005 Brokerage and Closure. Oxford University Press, Oxford.

Buskens, V.

2008 "Dynamics of Network if Everyone Strives for Structural Holes". American Journal of Sociology 114, 2:301-407.

Cáceres, J.

2007 Poder Rural y Estructura Social. Colchagua, 17601860. La Construcción del Estado y la Ciudadanía desde la Región. Universidad Católica de Valparaíso, Valparaíso.

Cariola, C. y Sunkel, O.

1982 La Historia Económica de Chile, 1830 y 1930: dos Ensayos y una Bibliografía. Instituto de Cooperación Interamericana, Madrid.

Caro, $\mathrm{R}$.

1982 The Path to Power. Alfred A. Knopf, New York.

Coleman, J.

1988 "Social capital in the creation of Human Capital". American Journal of Sociology 94: 95-120.

Collins, R.

1998 The Sociology of Philosophies. Mass, Harvard University Press, Cambridge.

Dalzell, R.

1987 Enterprising Elite. W.W. Norton, New York.

Di Maggio, $\mathrm{P}$.

1992 "Nadel's paradox revisited: relational and cultural aspects of organizational structure". En Network and Organizations, edited by N. Nohria and R. Eccles, pp. 118-142. Harvard Business School Press, Boston.

Fagan, J.

2017 How organizational turbulence shapes the broker vision advantage. Dissertation for the degree of Doctor of Philosophy, College of Business and Economics, University of Kentucky, Kentucky.

Goyal, S and F. Vega-Redondo, F.

2007 "Structural holes in social networks". Journal of Economic Theory 137, 1:460-492.

Granovetter, M.

1973 "The Strength of Weak Ties". American Journal of Sociology 78:1360-1380.

Kleinberg, J.; Suri, S; Tardos, É. and Wexler T.

2008 "Strategic network formation with structural holes". EC'08: 284-293.

Latora, V.; Nicosia, V.; Panzarasa, P.

2013 "Social cohesion, structural holes, and a tale of two measures”. Journal of Statistical Physics 151, 3-4:745-764.
Llorca, M.; Robles, C.; Navarrete-Montalvo, J.; Araya, R.

2017 "La agricultura y la élite agraria chilena a través de los catastros agrícolas, c.1830-1855”. Historia 50, II:597-639.

Lou, T.; Tang, J.

2013 "Mining structural hole spanners through information diffusion in social networks". Proceedings of the 22nd International Conference on World Wide Web, pp. 825-836, ACM, Rio de Janeiro.

Mellafe, R.

2004 Historia Social de Chile y América. Universitaria, Santiago.

Merton, R.

1957 "Pattern of influence: Local and cosmopolitan influentials". Social Theory and Social Structure 2: 441-474.

Muñoz, J.

2008 "La concesión de mercedes de tierra en la doctrina de Malloa (Colchagua, siglos XVI y XVII)". Espacio Regional 1, 5: 69-95.

Padgett, J.; Ansell, C.

1993 "Robust Action and the Rise of the Medici, 1400-1434". American Journal of Sociology 98:1259-1319.

Peeples, M.; Haas, R.

2013 "Brokerage and social capital in the prehispanic US Southwest". American Anthropologist 115,2: 232-247.

Rector, J.

1985 "El impacto económico de la independencia en América Latina: el caso de Chile. 1985". Historia 20:295-318.

Romano, R.

1965 Una Economía Colonial: Chile en el Siglo XVIII. Editorial Universitaria de Buenos Aires, Buenos Aires.

Salazar, G. y Pinto, J.

2002 Historia Contemporánea de Chile III. La Economía: Mercados, Empresarios y Trabajadores. LOM, Santiago.

Schumpeter, J.

1934 The Theory of Economic Development. Mass, Harvard University Press, Cambridge.

Tapia, A.; López, J. y Meseguer, O.

2018 "Capital Social de la comunidad de Timar, región de Arica y Parinacota, como recurso territorial para la adaptación ante perturbaciones ambientales". Diálogo Andino 55: 131-142.

Tortoriello, M.; Krackhardt, D.

2010 "Activating cross-boundary knowledge: the role of Simmelian ties in the generation of innovations". Acad. Manag 53, 1:167-181.

Travers, J.; Milgram, S.

1969 "An experimental study of the small world Phenomenon". Sociometry 32: 425-443.

\section{Fuentes Documentales}

Fondo de Notarios de Rengo. Volumen 2. Archivo Nacional Histórico.

Fondo de Notarios de Rengo. Volumen 26. Archivo Nacional de la Administración.

Fondo de Contaduría Mayor. Segunda serie, volumen 3742. Archivo Nacional Histórico.

Oficina Central de Estadística. 1855a. Rejistro Jeneral del Catastro Formado el año de 1852. Imprenta de J. Belín y Cía, Santiago.

Oficina Central de Estadística. 1855b. Estadística Agrícola de Chile. Imprenta del Diario La patria, Valparaíso. 


\section{Notas}

1 En todos los casos, la noción de Capital Social se comprende, como se interpretará en este estudio, como ventaja de los actores por obtención de recursos variados, en el contexto de sus interacciones sociales. De todos modos, existen lecturas de la noción de Capital Social que apuntan hacia el examen de la colaboración más que a la competencia de los actores por los recursos. En este sentido, resultan interesantes las investigaciones que abordan el Capital Social de colectividades, para, por ejemplo, adaptarse al medio ante coyunturas de cambio a distintas escalas (Tapia, et al. 2018).

2 En la selección del escenario de estudio emerge una pregunta adicional que complementa las que se abordan en este artículo: ¿Cómo se ajusta la teoría del capital social y de los agujeros estructurales, a un contexto "tradicional" o "precapitalista", considerando que aquel enfoque surgió para explicar redes en contextos industriales o postindustriales? La interrogante exhorta analizar otras redes contemporáneas (de parentesco, de poder local, etc.) en las que participaban los mismos actores, comprendiendo que los lazos sociales fueron más estrechos en este tipo de sociedades. Respecto del tópico de la superposición de redes sociales en contextos tradicionales y en perspectiva histórica, sigue resultando influyente el trabajo de John Padgett (1993) respecto de la movilización de intereses de Cosme de Medici en distintas redes sociales de Florencia durante el periodo renacentista. Debo al Dr. Vicente Espinoza el incentivo para pensar esta tensión entre teoría del brokerage y contexto tradicional. Abrimos esta discusión en el Coloquio Redes Sociales Interpersonales y Cohesión Social del Centre for Social Conflict and Cohesion Studies, en noviembre de 2018. Instancia a la que fui invitado a exponer por gentileza del Dr. Espinoza.

3 Víctor Brangier et al. (2018a) ha profundizado en la constitución de jueces legos en el valle central de Chile durante esta época. Particularmente ha examinado la pertenencia de alcaldes, subdelegados e inspectores a la comunidad de justiciables. También, la acumulación de atribuciones policiales, administrativas y judiciales en este tipo de agentes y el fundamento de su actuar procesal en las prácticas y representaciones sociales.

4 El examen de la información notarial fue complementada con la revisión de tres catastros agrícolas levantados, aproximadamente, en la época del estudio (1833/34; 1852 y 1855). Los documentos registran la rentabilidad agrícola anual de cada predio del país, para posteriormente fijar un tributo progresivo del $3 \%$ y posteriormente del $4 \%$. En la fuente se identifica el nombre del propietario y de su predio, información que propicia el cotejo con las escrituras notariales.

5 Debido a que los valores de las variables del estudio no presentan distribución normal entre sí (Efficient Size y Degree oscilan entre 1 y 16, mientras que los valores de Betweenness lo hacen entre 0 y 8.033,2), se utilizó el Test de Correlación de Spearman. El valor del coeficiente varía entre -1 y 1 . En la medida que el resultado se acerca a los extremos la correlación es fuerte, y, por el contrario, si se acerca a cero la relación es inexistente.

6 El rango del Coeficiente de Correlación se extiende de 1 a-1. Si se aproxima a los extremos la correlación es alta. Cifras iguales o mayores a 0,7 expresan correlación significativa.

7 Si el nivel de significancia en menor que 0,05 es significativo.

8 Los catastros agrícolas contemporáneos pretendieron gravar la rentabilidad anual de la producción agrícola destinada al mercado. Desde un principio, el mínimo consignado para ese gravamen fue de 12,4 pesos (Álvarez 2014).

9 Hemos estimado ese protagonismo entre $92 \%$ y $93 \%$ (Brangier 2019a).

10 Metodológicamente se ha recurrido al escalafón de rentabilidad informado por los catastros para diseñar las categorías de mediano y gran propietario. La primera categoría incluye propietarios agrícolas con rentabilidad entre el mínimo de 18,6 pesos y 100 pesos. Desde esa cifra en adelante se ha considerado como gran propietario (Brangier 2019b).

11 En esta batería de conductas puede incluirse la tendencia a concentrar los recursos en una sola red, evitando la diversificación de compras y ventas de tierra en otros sectores. Es una conclusión que deriva de la lectura de la columna "Localidad" de la Tabla 5: de los 10 brokers de rentabilidad media, solo dos registraron propiedades gravables en otras zonas. Los ocho restantes figuraron exclusivamente con terrenos en Pichidegua.

12 En un estudio reciente (Brangier et al. 2018b) hemos propuesto un estudio de caso de la trayectoria y estrategias de Vicente Echeñique, el principal broker de esta red. 
